\title{
Correction to: Different effects of $\gamma$-linolenic acid (GLA) supplementation on plasma and red blood cell phospholipid fatty acid composition and calcium oxalate kidney stone risk factors in healthy subjects from two race groups with different risk profiles pose questions about the GLA-arachidonic acid-oxaluria metabolic pathway: pilot study
}

\author{
Allen L. Rodgers ${ }^{1}$ - Dalielah Jappie-Mahomed ${ }^{1}$ • Paul J. van Jaarsveld ${ }^{2}$
}

Published online: 8 January 2019

(c) Springer-Verlag GmbH Germany, part of Springer Nature 2019

\section{Correction to: Urolithiasis (2018) 46:137-147}

https://doi.org/10.1007/s00240-017-0989-7

The authors would like to add the following paragraph in the acknowledgements section of the original version of the paper:

"The work described in this paper is based on studies performed by Pumeza Gogwana as part of her PhD thesis (Gogwana PC, 2014. Investigation of the potential beneficial effects of supplemental polyunsaturated fatty acids and glycosaminoglycans on the risk factors for calcium oxalate kidney stone formation using theoretical, experimental and human models. Doctoral dissertation, University of Cape Town). The authors duly acknowledge this contribution to the present paper".

So the Acknowledgements section should be:

The financial support of the South African Medical Research Council, the South African National Research Foundation and the University of Cape Town is gratefully acknowledged.

The original article can be found online at https://doi.org/10.1007/ s00240-017-0989-7.

Allen L. Rodgers

allen.rodgers@uct.ac.za

1 Department of Chemistry, University of Cape Town, Rondebosch, Cape Town 7701, South Africa

2 Non-Communicable Diseases Research Unit (NCDRU), South African Medical Research Council, Cape Town, South Africa
The authors would like to extend their appreciation to Johanna van Wyk of the NCDRU of the SAMRC for her invaluable laboratory assistance analyzing plasma and RBC total phospholipid fatty acids. The work described in this paper is based on studies performed by Pumeza Gogwana as part of her PhD thesis (Gogwana PC, 2014. Investigation of the potential beneficial effects of supplemental polyunsaturated fatty acids and glycosaminoglycans on the risk factors for calcium oxalate kidney stone formation using theoretical, experimental and human models. Doctoral dissertation, University of Cape Town). The authors duly acknowledge this contribution to the present paper. 